\title{
THERMAL STRUCTURE OF MAGNETIC FUNNEL FLOWS
}

\author{
STEVEN C. MARTIN \\ Department of Astronomy $\&$ Astrophysics \\ The University of Chicago \\ 5640 South Ellis Ave. \\ Chicago, Illinois 60637 \\ USA
}

\begin{abstract}
The thermodynamic structure of gas that is channeled by stellar magnetic fields onto a young (pre-main-sequence) star is presented. In this model, the star possesses a dipole magnetic field which disrupts the inner regions of a geometrically thin accretion disk and channels the inflowing gas onto the stellar surface, thereby forming an accretion funnel. The temperature and ionization degree of the inflowing gas is calculated by solving the heat equation coupled to statistical rate equations for hydrogen. It is found that for typical accretion rates of $\sim 10^{-7} M_{\odot} y r^{-1}$, temperatures of $\sim 7000 \mathrm{~K}$ and hydrogen ionization fractions $\left(n_{H^{+}} / n_{H}\right)$ of $\sim 10^{-2}$ can be attained in the funnel flow. The principal heat source is found to be adiabatic compression, and coolants include bremsstrahlung radiation as well as line emission from the Ca II and Mg II ions. The relatively hot and ionized funnel flow lead to observational signatures such as inverse P Cygni line profiles seen in upper Balmer and near-infrared lines. In addition, carbon monoxide bandhead emission may be an important tracer of the outer portions of the funnel flow.
\end{abstract}

\section{Introduction}

Many pre-main-sequence stars are known to have inflowing gas as well as outflowing gas. Both of these flows can manifest themselves simultaneously and appear to be physically related (e.g., Cabrit et al. 1990). Tracers of the outflows include P Cygni profiles of permitted lines (e.g., Mundt 1984), blueshifted forbidden emission lines (e.g., Hartigan, Edwards, \& Ghandour 1995), and well collimated optical jets which possess (many times multiple) 
Herbig-Haro objects (e.g., Mundt, Brugel, \& Bührke 1987). The inflowing gas is inferred to be in the form of a geometrically thin accretion disk (e.g., Adams, Lada, \& Shu 1988). Disk properties have been deduced by detailed modeling of both ultraviolet and infrared continuum emission (e.g., Bertout, Basri, \& Bouvier 1988; Hartigan, Edwards, \& Ghandour 1995).

In the event that the star is unmagnetized (or has a sufficiently weak magnetic field), the accretion disk joins onto the star through an equatorial boundary layer (e.g., Lynden-Bell \& Pringle 1974). However, in the presence of a strong stellar magnetic field, the inner accretion disk can become disrupted and the gas from the disk can be channeled onto the star along magnetic field lines forming accretion funnels (e.g., Königl 1991; Hartmann, Hewett, \& Calvet 1994). At the stellar surface, the infalling gas (essentially in free-fall) must be halted leading to strong shocks where the kinetic energy of the infalling gas is thermalized and re-radiated (mainly in the ultraviolet). This scenario may be ubiquitous among, for example, classical T Tauri stars as implied by rotating "hot spots" (e.g., Kenyon et al. 1994), emission line profiles (e.g., Edwards et al. 1994; and Edwards this volume), and inferred stellar magnetic field strengths (Basri, Marcy, \& Valenti 1992, see also Guenther this volume).

The radiative properties of magnetic funnel flows are determined by both the kinematic and thermodynamic characteristics of the infalling gas. By solving the thermal balance equation coupled to rate equations for hydrogen, the temperature and ionization structure for funnel flows can be computed. These calculations can form the basis for interpreting the observed tracers of funnel flows from young stars, in particular P Cygni line profiles and possibly carbon monoxide bandhead emission.

\section{Gas Dynamics}

To compute the dynamics of the gas, the star is assumed to possess a dipole magnetic field which disrupts a geometrically thin accretion disk at several stellar radii (typically at $\sim 5$ stellar radii). The entire flow is therefore axisymmetric and specified a priori. Although the geometry adopted here is dipolar, the calculations and results would be valid for generic converging inflows (the model would also be applicable even if the field were not continuous, e.g., magnetic flux tubes). Since this model is most appropriate for $\mathrm{T}$ Tauri stars, rotation is not important on the dynamics of the infalling gas (T Tauri stars are slow rotators, see Hartmann \& Stauffer 1989).

In general, the inflowing material consists of several components: neutral gas, ionized gas, and dust. If the neutrals were not well coupled to the ions through collisions, the neutrals would fall toward the star along radial trajectories while the ions would follow the magnetic field lines. At the other 
extreme, perfect ion-neutral coupling would ensure that both components flow inward along the magnetic field lines. Using the equation of motion for the neutrals, it is possible to estimate the relative drift between these two components and therefore assess how good the coupling is (see Martin 1996). It turns out the coupling is sufficient throughout the flow to treat the ions and neutrals as effectively coupled. Dust could have strong effects both on the emergent radiation from the funnel flow and on its thermodynamic structure. However, it is most likely that funnel flows are dust free based on the small values of $A_{v}(\sim$ few at most) deduced for T Tauri stars (see Appendix A of Martin 1996). Thus, the inflowing material can be treated as a single fluid and both the velocity and density of the gas can be derived at any point along the flow (see Hartmann, Hewett, \& Calvet 1994).

\section{Gas Thermodynamics}

The temperature and ionization degree of the gas can be determined by solving the first law of thermodynamics coupled to rate equations for hydrogen (Martin 1996). The first law reduces to the standard heat equation in steady state (in a fixed reference frame) and can be written as

$$
\frac{3}{2} n_{\mathrm{H}} k v \cdot \nabla T=k T \boldsymbol{v} \cdot \nabla n_{\mathrm{H}}-n_{\mathrm{H}} \sum_{j} I_{j} v \cdot \nabla f_{j}+\Gamma-\Lambda,
$$

where $n_{\mathrm{H}}$ is the total number density of hydrogen nuclei, $T$ is the kinetic temperature, $I_{j}$ is the binding energy of hydrogen in state $j, f_{j}$ is the fractional particle density $\left(n_{j} / n_{H}\right)$ and $k$ is Boltzmann's constant. Note the meaning of the terms on the right hand side of equation (1): the first term represents adiabatic cooling for an expanding flow and compressional heating for a converging flow, the second term allows for changes in the chemistry along the flow, the third term $(\Gamma)$ is the heating rate per unit volume and the last term $(\Lambda)$ is the cooling rate per unit volume. In practice, the most difficult part of solving equation (1) is specifying the last two terms for the densities and temperatures of interest in the funnel flow (typically, $T \sim 5000 \mathrm{~K}$ and $n_{H} \sim 10^{12} \mathrm{~cm}^{-3}$ for an accretion rate $\sim 10^{-7} M_{\odot} y r^{-1}$ ). The required rate equations for hydrogen may be written simply as

$$
\boldsymbol{v} \cdot \nabla f_{j}=\sum_{j^{\prime} \neq j} R_{j^{\prime}, j}-f_{j} \sum_{j^{\prime} \neq j} R_{j, j^{\prime}}
$$

where $R$ is the creation or destruction rate $\left(\mathrm{s}^{-1}\right)$ due to collisional and radiative processes. This system of equations is closed by requiring particle conservation. Once the model parameters are specified (e.g., stellar mass, radius, and temperature; the mass accretion rate; and the initial temperature) the above system of equations is integrated from a point just above 

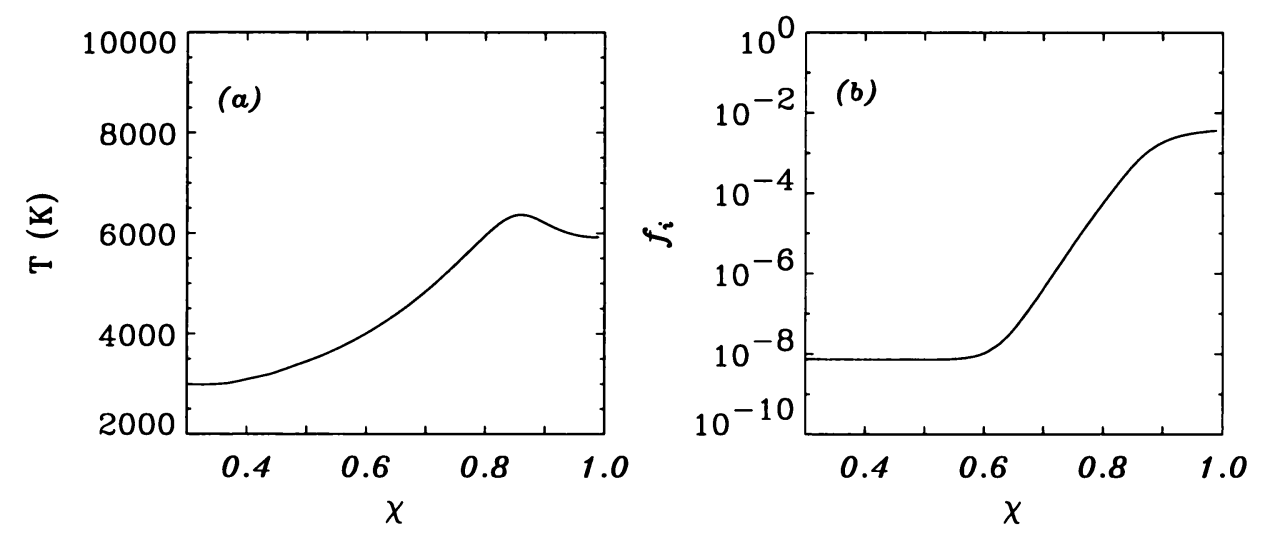

Figure 1. Thermal structure of gas along a flow line for parameters of a T Tauri star and a mass accretion rate of $10^{-7} M_{\odot} y r^{-1}$. The abscissa is the fraction along the flow line measured in units of total flow line length. The equations are integrated from $\chi=0.3$ to $\chi \approx 1$ where the shock forms. (a) Temperature and (b) ionization fraction $f_{i}=n_{H^{+}} / n_{H}$ (from Martin 1996).

the disk (where the gas is loaded onto the field lines) to the stellar surface. The results from a sample calculation are displayed in Figure 1. Near the end point of the flow (i.e., the shock at $\chi \approx 1$ ), the largest temperature and ionization degree is attained. The high densities encountered in the funnel flow prevent Lyman photons from effectively penetrating and ionizing the gas. However, Balmer continuum photons can easily penetrate the gas flow and are the main ionizing source. The heat sources and coolants for this calculation can be identified from Figure 2. The principal heat source is compressional, due to the converging nature of the flow. This heating leads to a continual rise in temperature and ionization degree until $T \sim 6500 \mathrm{~K}$ is reached, at which point the $\mathrm{Ca}$ II and $\mathrm{Mg}$ II ions efficiently cool the gas. In fact, these ions behave as a thermostat that regulates the gas temperature (see Appendix B of Martin 1996).

\section{Observational Implications}

Recent observations of young stars have found several pieces of evidence for magnetically channeled gas accretion (e.g., Kenyon et al. 1994, Edwards et al. 1994, and see especially Edwards this volume). Emission line profiles provide the most useful diagnostics for these types of flows as they give information on both the gas kinematics and thermodynamics. Inverse $\mathrm{P}$ Cygni line profiles were first recognized in some of the upper Balmer lines (Edwards et al. 1994), but now lines in the infrared (e.g., $\mathrm{Pa} \beta$ ) are routinely 

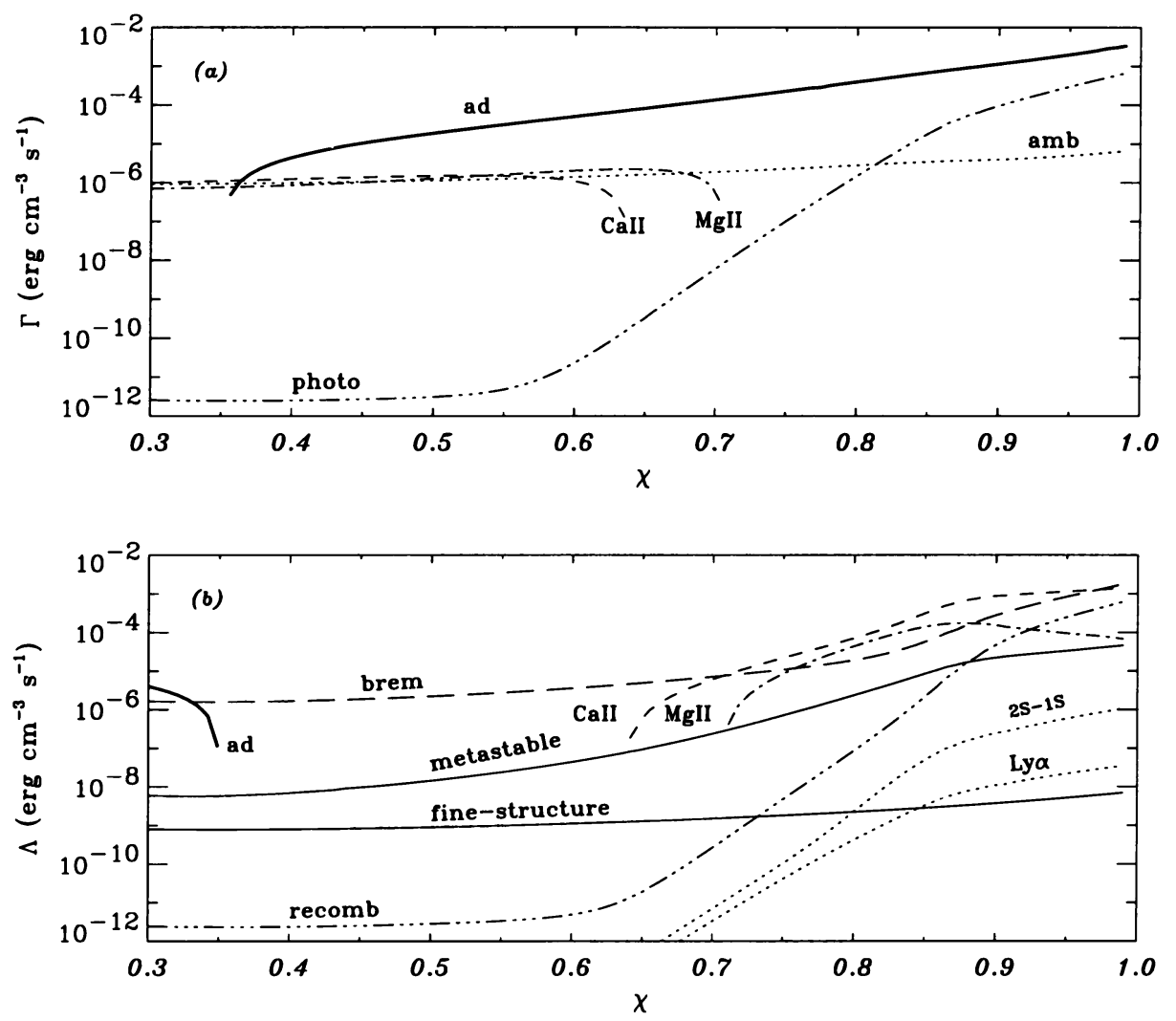

Figure 2. Heating and cooling rates for the calculation shown in Figure 1. (a) Heating rates. Heavy solid line: adiabatic; dotted line: ambipolar diffusion. (b) Cooling rates. Heavy solid line: adiabatic; long dashed line: bremsstrahlung; short dashed line: Ca II; dash-dotted line: Mg II; triple-dot dashed line: recombination. See Martin (1996) for details.

being observed (Folha, Emerson, \& Calvet 1997). In addition, since some regions of the funnel flow are cool enough to allow molecules to exist (e.g., carbon monoxide), they may provide another important tracer of funnel flows.

The highest temperatures and ionization degrees are found in the inner regions of the funnel flow (see Fig. 1). For a typical accreting T Tauri star, temperatures $\sim 6500 \mathrm{~K}$ and ionization fractions of a few percent can be established. Thus, this model predicts that inverse $P$ Cygni line profiles seen in the higher Balmer lines should form in the inner flow regions, where the temperatures are sufficient to populate the upper levels. The kinematics of the flow can be understood from Figure 3 . This figure displays the constant- 


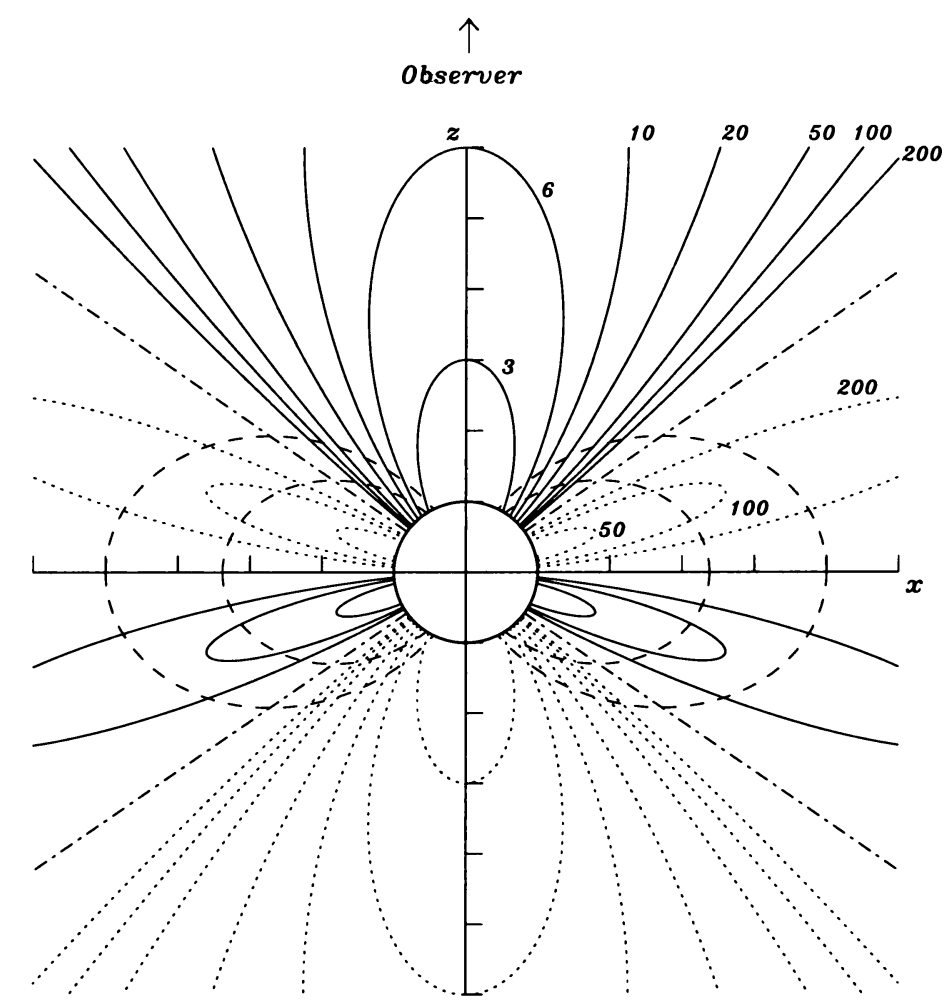

Figure 3. Constant-projected-velocity curves for an inclination of $0^{\circ}$ in a dipole flow geometry. The Curves are labeled by $\Upsilon=\left(v_{f f} / v_{l o s}\right)^{2}$ where $v_{f f}$ is the free-fall velocity and $v_{l o s}$ is the line-of-sight velocity. Positive velocities (redshifted) are represented by solid curves and negative velocities (blueshifted) by dotted curves (Martin 1996).

projected-velocity curves in a dipole flow geometry. The largest velocities occur in the inner regions (near the shock) and thus redshifted absorption within a line would occur at relatively large velocities. This prediction is borne out by observations where redshifted absorption features are seen at velocities $\gtrsim 200 \mathrm{~km} \mathrm{~s}^{-1}$. Furthermore, blueshifted absorption features (at times seen simultaneously) typically occur at velocities $\lesssim 100 \mathrm{~km} \mathrm{~s}^{-1}$ and are most likely due to absorption in an outflowing wind.

Molecular lines, such as the first overtone transitions of carbon monoxide $(\mathrm{CO})$, probe moderate-temperature $(T \sim 3000 \mathrm{~K})$, high-density $\left(n_{H} \gtrsim\right.$ 


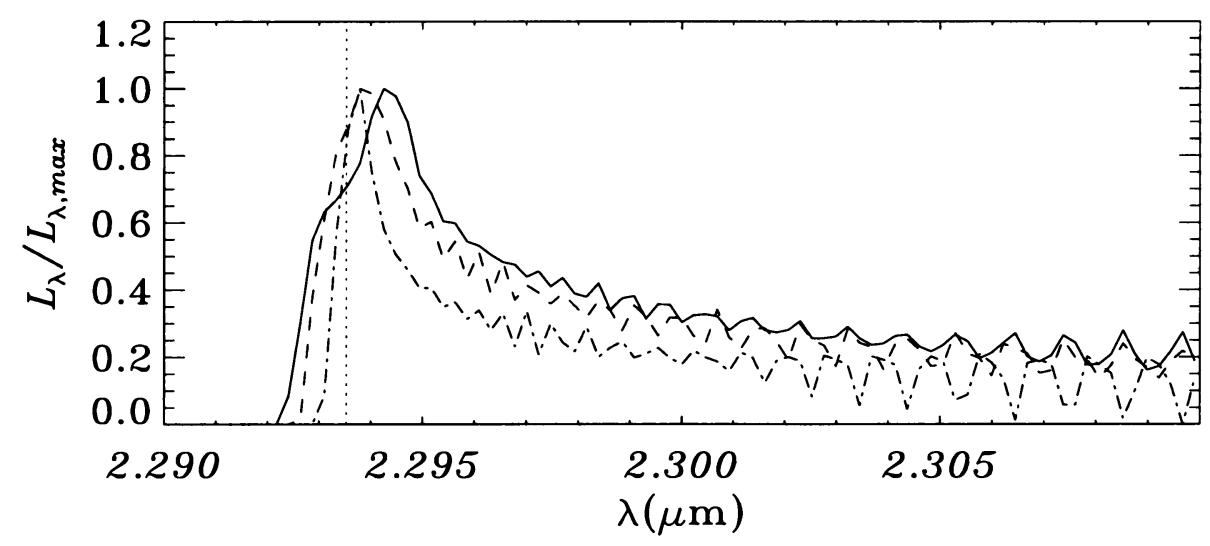

Figure 4. Bandhead profiles of the $v=2-0$ transition of $\mathrm{CO}$ for the thermodynamic model shown in Figure 1. The solid line, dashed line, and dash-dotted line refer to viewing angles (with respect to the rotation axis) of $80^{\circ}, 45^{\circ}$, and $10^{\circ}$, respectively. Dotted line: rest wavelength of the bandhead. From Martin 1997.

$10^{10} \mathrm{~cm}^{-3}$ ) gas. These conditions can be found in funnel flows and thus $\mathrm{CO}$ could be an important probe of the outer regions. High-resolution line profiles of $\mathrm{CO}$ bandheads from young stars indicate emission up to relatively large velocities ( $\pm \sim 200 \mathrm{~km} \mathrm{~s}^{-1}$; e.g., Chandler et al. 1993). These profile characteristics have led most authors to favor an accretion disk as the origin of the CO lines (e.g., Chandler, Carlstrom, \& Scoville 1995). However, it turns out that in a dipole flow geometry the kinematics and thermodynamics in the outer portions of the funnel flow can give the observed line profiles (Martin 1997). Shown in Figure 4 are a sample of the $v=2-0$ bandhead profiles for $\mathrm{CO}$ using the computed thermal structure shown in Figure 1. Hence, the observed features (such as the blue shoulder and the redshifted peak) in this model depend mainly on the observers inclination angle. In addition, the computed luminosities of the bandheads fall near the low end of the observed range. Since the luminosity is directly proportional to the density and therefore the mass accretion rate (see Martin 1997), only those sources with the largest accretion rates should have detectable CO bandhead emission.

\section{Conclusions}

In the funnel flow model, stellar magnetic field lines disrupt the innermost regions of an accretion disk and channel the gas onto the surface at relatively high latitudes (see Figure 5). Since the flow is converging, compressional heating operates and increases the gas temperature to values $\sim 7000 \mathrm{~K}$. At 


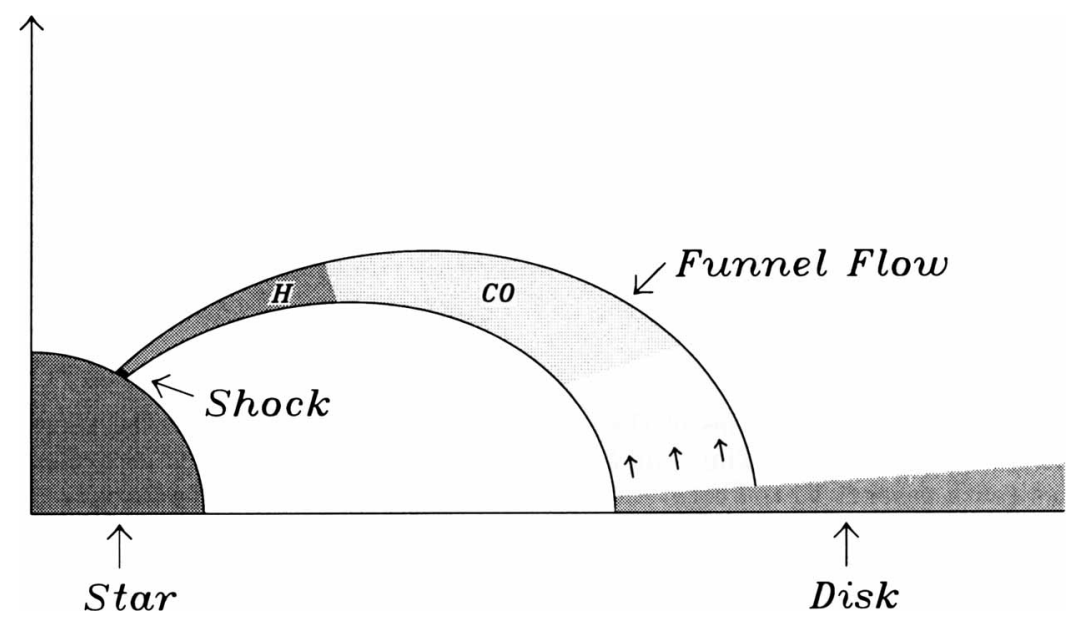

Figure 5. Dipolar funnel flow model. Only one quadrant is displayed, since the model is axisymmetric and reflection symmetric. Temperature increases towards the shock. Light-shaded region represents the portion of the flow that can produce carbon monoxide bandhead emission. Dark-shaded region is where inverse P Cygni profiles form (e.g., higher Balmer lines). From Martin 1997.

the stellar surface, strong shocks form where the gas in free-fall is halted and its kinetic energy thermalized, leading to excess ultraviolet radiation. Many pre-main-sequence stars display signatures of gas inflow along stellar magnetic field lines. For example, some T Tauri stars display inverse $\mathrm{P}$ Cygni line profiles and ultraviolet excess. The kinematics and thermodynamics of the infalling gas imply that inverse P Cygni line profiles (of, e.g., hydrogen lines) form close to the accretion shock, where the gas velocities are largest. In fact, the observed profiles display absorption at relatively large redshifted velocities, as expected from the model. In addition, the outer regions of the flow are cool enough to allow molecular emission, and thus may explain the carbon monoxide bandhead profiles seen for some young stars.

Acknowledgements: The author would like to thank the symposium organizers for the invitation to present this work. This research was supported in part by NASA grants NAG5-2766 and NAG5-3687. Also, funding to attend this symposium was provided in part by a AAS international travel grant and an IAU travel grant. 


\section{References}

Adams, F. C., Lada, C. J., \& Shu, F. H. 1988, ApJ, 326, 865

Basri, G., Marcy, G. W., \& Valenti, J. A. 1992, ApJ, 390, 622

Bertout, C., Basri, G., \& Bouvier, J. 1988, ApJ, 330, 350

Cabrit, S., Edwards, S., Strom, S. E., \& Strom, K. M. 1990, ApJ, 354, 687

Chandler, C. J., Carlstrom, J. E., Scoville, N. Z., Dent, W. R. F., \& Geballe, T. R. 1993, ApJ, 412, L71

Chandler, C. J., Carlstrom, J. E., \& Scoville, N. Z. 1995, ApJ, 446, 793

Edwards, S., Hartigan, P., Ghandour, L., \& Andrulis, C. 1994, AJ, 108, 1056

Folha, D., Emerson, J., \& Calvet, N. 1997, in Low Mass Star Formation - from Infall to Outflow, poster proceedings of IAU Symp. No. 182, eds. F. Malbet \& A. Castets, p. 272

Hartigan, P., Edwards, S., \& Ghandour, L. 1995, ApJ, 452, 736

Hartmann, L., Hewett, R., \& Calvet, N. 1994, ApJ, 426, 669

Hartmann, L., \& Stauffer, J. R. 1989, AJ, 97, 873

Kenyon, S. J., Hartmann, L., Hewett, R., Carrasco, L., Cruz-Gonzalez, I., Recillas, E., Salas, L., Serrano, A., Strom, K. M., Strom, S. E., \& Newton, G. 1994, AJ, 107, 2153

Königl, A. 1991, ApJ, 370, L39

Lynden-Bell, D., \& Pringle, J. E. 1974, MNRAS, 168, 603

Martin, S. C. 1996, ApJ, 470, 537

. 1997, ApJ, 478, L33

Mundt, R. 1984, ApJ, 280, 749

Mundt, R., Brugel, E. W., \& Bührke, T. 1987, ApJ, 319, 275 